\title{
BMJ Open Association between intrahepatic triglyceride content in subjects with metabolically healthy abdominal obesity and risks of pre-diabetes plus diabetes: an observational study
}

\author{
Qiaoyan Xu, ${ }^{1}$ Junfeng Zhang, ${ }^{1}$ Haiwei Han, ${ }^{2}$ Ning Chen $(1),{ }^{3}$ Fangfang Lai, ${ }^{1}$ \\ Yongwen Liu, ${ }^{4}$ Caoxin Huang, ${ }^{5}$ Mingzhu Lin, ${ }^{6}$ Wei Zhang, ${ }^{7}$ Shunqin Wang, ${ }^{8}$ \\ Changqin Liu (1) , ${ }^{6,9}$ Zhibin $\mathrm{Li}^{10}$
}

To cite: Xu Q, Zhang J, Han H, et al. Association between intrahepatic triglyceride content in subjects with metabolically healthy abdominal obesity and risks of prediabetes plus diabetes: an observational study. BMJ Open 2022;12:e057820. doi:10.1136/ bmjopen-2021-057820

- Prepublication history for this paper is available online. To view these files, please visit the journal online (http://dx.doi. org/10.1136/bmjopen-2021057820).

$\mathrm{QX}, \mathrm{JZ}, \mathrm{HH}$ and NC contributed equally.

Received 29 September 2021 Accepted 18 January 2022

Check for updates

(c) Author(s) (or their employer(s)) 2022. Re-use permitted under CC BY-NC. No commercial re-use. See rights and permissions. Published by BMJ.

For numbered affiliations see end of article.

\section{Correspondence to}

Dr Changqin Liu;

liuchangqin@xmu.edu.cn and

Dr Zhibin Li;

zhibinli33@hotmail.com

\section{ABSTRACT}

Objective We aimed to evaluate the association of intrahepatic triglyceride (IHTG) content in subjects with metabolically healthy abdominal obesity (MHAO) on risks of pre-diabetes plus diabetes.

Design Cross-sectional survey.

Setting Lianqian community, the First Affiliated Hospital of Xiamen University, Xiamen, China.

Participants Among 1523 community-living healthy adults aged 40 years or older with abdominal obesity recruited at baseline, 428 subjects who underwent IHTG content measurement were selected.

Outcome measures Risk of pre-diabetes plus diabetes. Results Non-alcoholic fatty liver disease (NAFLD) was diagnosed as 203 (69.1\%) in MHAO and 121 (90.3\%) in metabolically unhealthy abdominal obesity (MUAO) $(p<0.001)$. The prevalence rates of pre-diabetes plus diabetes were $81.1 \%, 88.8 \%$ and $90.9 \%$ across the tertiles of IHTG content ( $p=0.037)$. Both MUAO (vs MHAO) and NAFLD (vs non-NAFLD) were independently associated with increased risks of pre-diabetes plus diabetes, the adjusted ORs (95\% Cls) were 10.90 (3.15 to 37.69 , $\mathrm{p}<0.001$ ) and 3.02 (1.47 to $6.20, \mathrm{p}=0.003$ ), respectively. Higher IHTG content was significantly associated with increased risk of pre-diabetes plus diabetes with the adjusted $\mathrm{OR}(95 \% \mathrm{Cl})$ of per SD increase of IHTG content of 1.62 ( 1.07 to $2.46, p=0.024$ ). And there was a significantly positive trend between increasing categories of IHTG content tertiles and excessive risks of pre-diabetes plus diabetes (trend test $p$ value $=0.011$ ). Stratified analyses showed similar results on the associations of NAFLD and IHTG content with risks of pre-diabetes plus diabetes for subjects with MHAO but not for those with MUAO.

Conclusions NAFLD and higher IHTG content were independently associated with increased risks of prediabetes plus diabetes in MHAO subjects. NAFLD or quantity of liver fat should be considered as additional criterion when defining and diagnosing $\mathrm{MHO}$. Screening of NAFLD and intervention to reduce liver fat should be strengthened even for those seemly metabolically healthy obese.
Strengths and limitations of this study

This study was a cross-sectional analysis of baseline information on the ongoing cohort study to evaluate the independent association of intrahepatic triglyceride (IHTG) content with risk of pre-diabetes plus diabetes.

- All subjects were abdominally obese and were not randomly sampled from their living communities.

- IHTG content was determined using magnetic resonance spectroscopy which was relatively quantitative measurement of liver fat.

- The sample size was relatively small, especially for the metabolically unhealthy abdominal obesity subgroup, and we might not have enough power to determine their true associations.

\section{INTRODUCTION}

The prevalence of diabetes has quadrupled during the past three decades with an estimated prevalence of $9.3 \%$ (463 million people) in 2019 and it is expected to rise to $10.2 \%$ (578 million) by 2030 and $10.9 \%$ (700 million) by $2045 .^{1-3}$ Obesity has been well documented to be a risk factor for a broad array of chronic non-communicable diseases, including diabetes, hypertension, coronary heart disease, chronic kidney disease and certain sites of cancer. ${ }^{4-6} \mathrm{~A}$ subgroup of individuals with obesity who are devoid of obesity-related metabolic complications, such as diabetes and atherosclerosis, arise the concept of metabolically healthy obese (MHO) ${ }^{7-9}$ However, there is no unique definition and diagnose criteria for $\mathrm{MHO}$ by now. For example, some defined MHO when two or fewer of the four criteria of metabolism syndrome $^{10}$ for those subjects with obesity while others defined as none of them, ${ }^{11}$ which 
1523 residents aged 40 years or older with central obesity (waist circumference $\geq 90 \mathrm{~cm}$ for men and $80 \mathrm{~cm}$ for women) in Lianqian community, Xiamen, China

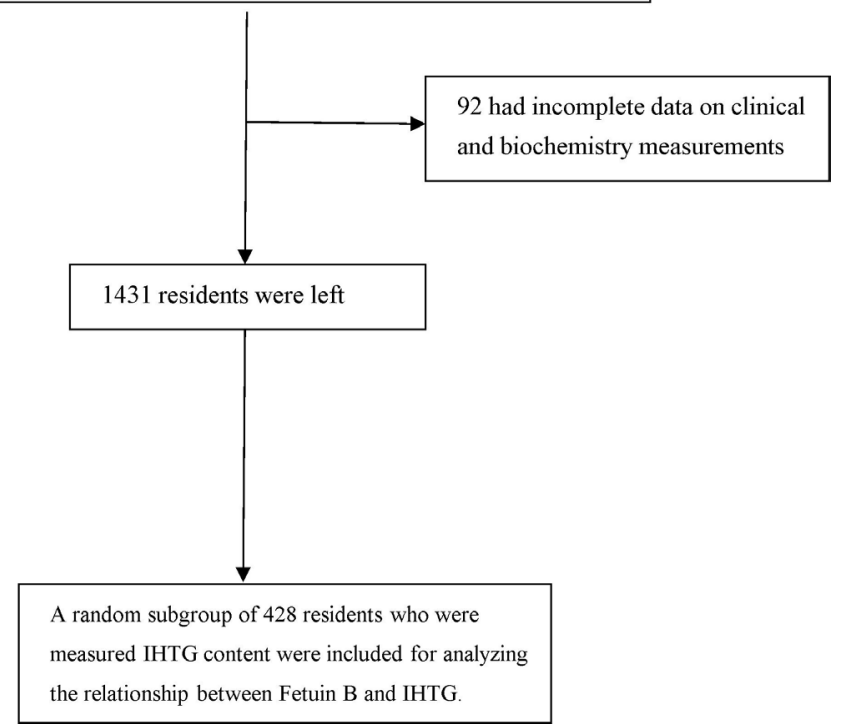

Figure 1 Study subjects' selection diagram. IHTG, intrahepatic triglyceride.

made evidence on the association of $\mathrm{MHO}$ with diabetes was limited and controversial. ${ }^{12}$

Non-alcoholic fatty liver disease (NAFLD) is well documented to be associated with risk of diabetes ${ }^{13}$; however, NAFLD has not been considered as additional criterion for MHO although it usually occurs simultaneously when obesity happens. Therefore, little evidence is available on the risk of NAFLD or liver fat with diabetes for those with MHO. In the present study with 428 communityliving Chinese adults with abdominal obesity, we mainly aimed to evaluate associations of intrahepatic triglyceride (IHTG) content and NAFLD in subjects with metabolically healthy abdominal obesity (MHAO) on risks of prediabetes plus diabetes.

\section{METHODS}

\section{Study design and subjects}

Details on study design and subject recruitment have been described previously. ${ }^{14-16}$ Briefly, 1523 communityliving healthy adults aged 40 years or older with abdominal obesity (waist circumference greater than $90 \mathrm{~cm}$ for men and $80 \mathrm{~cm}$ for women) living in Lianqian community, Xiamen, China were recruited at baseline of the cohort study in 2011. Of them, $92(6 \%)$ who had incomplete data on clinical and biochemistry measurements were excluded, and a random sample of 428 subjects who underwent IHTG content measurement was left for the present analysis (figure 1). Of the 428 study subjects, 319 $(74.5 \%)$ were female with the mean age of $53.6 \pm 6.5$ years old, $109(25.5 \%)$ were male with the mean age of $53.2 \pm 7.1$ years old, and there was no significant difference in age between male and female subjects $(p=0.592)$.

\section{Measurements}

Details on study measurements have been described previously. ${ }^{1516}$ For each subject, face-to-face interview was conducted to collect sociodemographic status, lifestyle habits, present and previous history of health and medications. Subjects were excluded if they drank regularly with alcohol consumption $\geq 140 \mathrm{~g} /$ week for men or $\geq 70$ $\mathrm{g} /$ week for women, had cancer, or received current treatment with systemic corticosteroids, biliary obstructive diseases, acute or chronic virus hepatitis, drug-induced liver diseases, total parenteral nutrition, autoimmune hepatitis, Wilson's disease, known hyperthyroidism or hypothyroidism. Subjects underwent weight, height and waist circumference measurements by using a calibrated scale after removing shoes and heavy clothes. Waist circumference was measured at the midpoint between the inferior costal margin and the superior border of the iliac crest on the midaxillary line. Body mass index (BMI) was calculated as weight in kilograms divided by height in squared metres. Arterial blood pressure (BP) was measured with a mercury sphygmomanometer after sitting for at least $15 \mathrm{~min}$.

Blood samples were obtained after 12-hour fasting and $75 \mathrm{~g}$ oral glucose tolerance test were conducted for each subject. All biochemical measurements were tested in the central laboratory of the First Affiliated Hospital, Xiamen University. Plasma glucose and serum lipid profiles, including triglyceride (TG), total cholesterol (TC) and high-density lipoprotein cholesterol (HDL-C) were determined on a HITACHI 7450 analyzer (HITACHI, Tokyo, Japan). Low-density lipoprotein cholesterol (LDL-C) was calculated by Friedewald's formula. Fasting plasma glucose (FPG) and 2-hour plasma glucose (2-h PG) concentrations were measured by the hexokinase method and Hemoglobin A1c (HbA1c) by the Bio-Rad Variant Hemoglobin A1c assay.

\section{Ultrasonography and definition of NAFLD}

Details on ultrasonography and definition of NAFLD have been described previously. ${ }^{15}{ }^{16}$ Hepatic ultrasonography scanning was performed by an experienced radiologist using GE LOGIQ P5 scanner (GE Healthcare, Milwaukee, USA) with a 4-MHz probe, who was blinded to the subjects' health status. Hepatic steatosis was diagnosed on the basis of characteristic sonographic features, including hepatorenal echo contrast, liver parenchymal brightness, deep beam attenuation and vessel blurring. ${ }^{17}$ The definition of NAFLD was based on hepatic ultrasonography diagnosis of hepatic steatosis without excessive alcohol consumption, viral or autoimmune liver disease. ${ }^{1516}$

\section{IHTG content measurement}

Details on IHTG content measurement has been described previously. ${ }^{18}$ IHTG content was determined by an experienced radiologist using magnetic resonance spectroscopy $\left({ }^{1} \mathrm{H}\right.$ MRS, 3.0-T Avanto, Siemens AG, Erlangen, German). Images of a sagittal, coronal and axial cube of 
a $2 \mathrm{~cm}^{3}$ volume in the right lobe of liver was acquired. Quantification of the spectra (water and methylene resonances) was performed as described previously. ${ }^{19}$ Areas of resonance from water protons and methylene groups in fatty acid chains were obtained with a time-domain non-linear fitting routine by using Syngo MR B15V software (Siemens AG). The percentage of IHTG content was calculated as the ratio of the area under the resonance of peak for methylene groups in fatty acid chains of IHTG and the combined area under the resonance peaks for both methylene groups and water. ${ }^{18} 19$

\section{Definition of MHAO}

Abdominal obesity was defined as $\mathrm{WC} \geq 90 \mathrm{~cm}$ for men and $80 \mathrm{~cm}$ for women. ${ }^{20}$ All subjects in the present study were abdominal obesity which was considered as one of the recruitment criteria. Subjects were defined as MHAO if two or fewer of the following criteria were met: (1) systolic $\mathrm{BP} \geq 130$ or diastolic $\mathrm{BP} \geq 85 \mathrm{mmHg}$; (2) $\mathrm{FPG} \geq 100 \mathrm{mg}$ / $\mathrm{dL}(5.6 \mathrm{mmol} / \mathrm{L})$; (3) $\mathrm{TG} \geq 150 \mathrm{mg} / \mathrm{dL}(1.7 \mathrm{mmol} / \mathrm{L})$; (4) HDL cholesterol $<40 \mathrm{mg} / \mathrm{dL}(1.03 \mathrm{mmol} / \mathrm{L})$ in men and $<50 \mathrm{mg} / \mathrm{dL}(1.30 \mathrm{mmol} / \mathrm{L})$ in women. ${ }^{16} 2122$ Otherwise, subjects meeting 3 or more of the criteria were defined as metabolically unhealthy abdominal obesity (MUAO). Therefore, all subjects in the present study were dichotomised as either MHAO or MUAO.

\section{Definitions of diabetes and pre-diabetes}

According to American Diabetes Association 2020 criteria, diabetes was defined as (1) a self-reported history of diabetes previously diagnosed by healthcare professionals; (2) FGP $\geq 126 \mathrm{mg} / \mathrm{dL}(7.0 \mathrm{mmol} / \mathrm{L})$; (3) 2-hour plasma glucose (2-h PG, oral glucose tolerance test $) \geq 200$ $\mathrm{mg} / \mathrm{dL}(11.1 \mathrm{mmol} / \mathrm{L})$; or (4) HbAlc $\geq 6.5 \%$. Pre-diabetes was defined as (1) FPG levels between $100 \mathrm{mg} / \mathrm{dL}$ (5.6 $\mathrm{mmol} / \mathrm{L})$ and $125 \mathrm{mg} / \mathrm{dL}(6.9 \mathrm{mmol} / \mathrm{L})$, (2) 2-h PG levels between $140 \mathrm{mg} / \mathrm{dL}(7.8 \mathrm{mmol} / \mathrm{L})$ and $199 \mathrm{mg} / \mathrm{dL}$ $(11.0 \mathrm{mmol} / \mathrm{L})$, or $(3) \mathrm{HbA} 1 \mathrm{c}$ between $5.7 \%$ and $6.4 \%$ in participants without a prior diabetes diagnosis. ${ }^{15} 1623$

\section{Statistical analyses}

Methods on statistical analyses were similar to our previous publications. ${ }^{151618}$ Data were presented as the mean \pm SD for continuous variables or number and percentage for categorical variables. Skewness and kurtosis tests for continuous variables were conducted and found them followed approximation of normal distributions. Differences between subjects categorised by MHAO and tertiles of IHTG content were analysed using one-way analysis of variance for continuous variables and $\mathrm{X}^{2}$ test for categorical variables. Bar graphs showing prevalence rates of diabetes, pre-diabetes and normal glucose test (NGT) were made by MHAO (vs MUAO) and tertiles of IHTG content.

Multivariable logistic regression models were used to calculate the adjusted ORs and 95\% CIs of abdominal obesity (MUAO vs MHAO), NAFLD (yes vs no) and IHTG content (both the originally continuous values and the tertiles categories) for pre-diabetes plus diabetes with adjustment for potential confounders (including age, sex, educational level, smoking and drinking habits, regular physical exercise, BMI, systolic and diastolic BP, TG, TC, HDL-C and LDL-C and serum uric acid). And multivariable logistic regression analyses stratified by MHAO and MUAO groups were further conducted. All $\mathrm{p}$ values were two sided and $p$ value $<0.05$ was considered statistically significant. All statistical analyses were performed using Stata V.14.0 (StatCorp).

\section{Patient and public involvement}

There were no funds or time allocated for patient and public involvement.

\section{RESULTS}

Prevalence of diabetes and pre-diabetes stratified by MHAO and tertiles of IHTG content

Among the 428 subjects with abdominal obesity, MHAO and MUAO were identified on $294(68.7 \%)$ and 134 $(31.3 \%)$ subjects. Of them, $46(10.8 \%), 326(76.2 \%)$ and $56(13.1 \%)$ were diagnosed as diabetes, pre-diabetes and NGT, respectively. There was a significantly positive trend between increasing tertiles of IHTG content and higher prevalence of pre-diabetes plus diabetes $(81.1 \%, 88.8 \%$ and $90.9 \%$ across the tertiles of IHTG content $(\mathrm{p}=0.037))$. Figure 2A showed the prevalence rates of diabetes and pre-diabetes across the tertiles of IHTG content in MHAO subjects were $7.1 \%$ and $67.3 \%, 10.2 \%$ and $74.5 \%, 10.2 \%$ and $77.6 \%$ for the tertile 1 , tertile 2 and tertile 3 , respectively ( $\mathrm{p}$ value $>0.05$ ). But there was a significantly positive trend of higher prevalence of diabetes plus diabetes with increasing categories of tertiles of IHTG content $(p=0.039)$. Figure 2B showed the prevalence rates of diabetes and pre-diabetes across the tertiles of IHTG content in MUAO subjects were $8.9 \%$ and $86.7 \%, 8.9 \%$ and $88.9 \%, 25.0 \%$ and $72.7 \%$ for tertile 1, tertile 2 and tertile 3, respectively. Table 1 also showed MUAO subjects had significantly higher prevalence of pre-diabetes and pre-diabetes plus diabetes than MHAO subjects (both $\mathrm{p}$ values $<0.05)$.

\section{Demographic and clinical characteristics stratified by MHAO and tertiles of IHTG content}

For all the 428 subjects, the means $( \pm \mathrm{SD})$ of age were 53.6 $( \pm 6.5)$ years for women $(\mathrm{n}=319,74.5 \%)$ and $53.2( \pm 7.1)$ years for men $(\mathrm{n}=109,25.5 \%)(\mathrm{p}=0.592)$. Table 1 showed differences of demographics, life style habits and clinical characteristics stratified by MHAO and tertiles of IHTG content. For 294 MHAO subjects, with increasing categories of the tertiles of IHTG content (from tertile 1, tertile 2 to tertile 3 ), subjects were more likely to be male and had significantly higher levels of indices of obesity (BMI, waist circumference), diastolic BP, TG, HbA1c, serum uric acid as well as higher prevalence of NAFLD and significantly lower level of HDL-C. As for 134 MUAO subjects, increasing categories of the tertiles of IHTG content were 
A

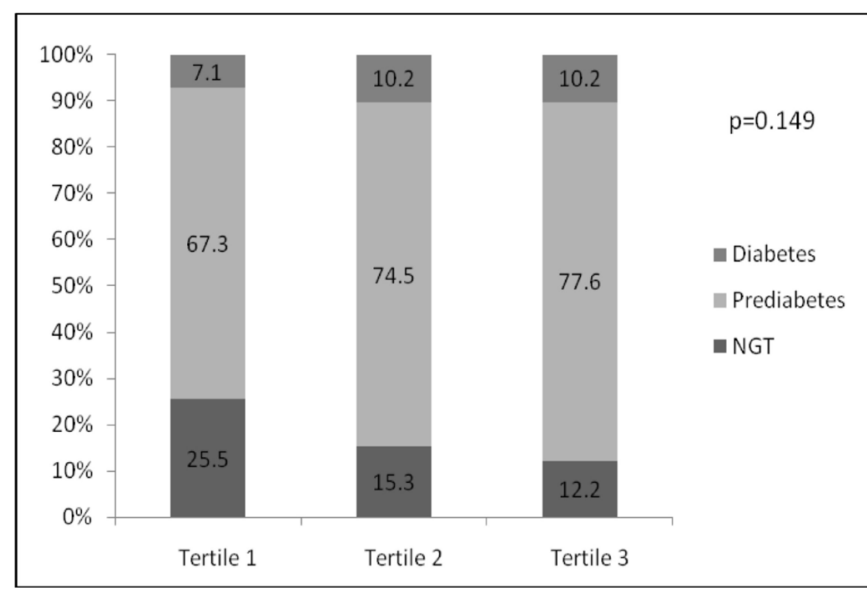

B

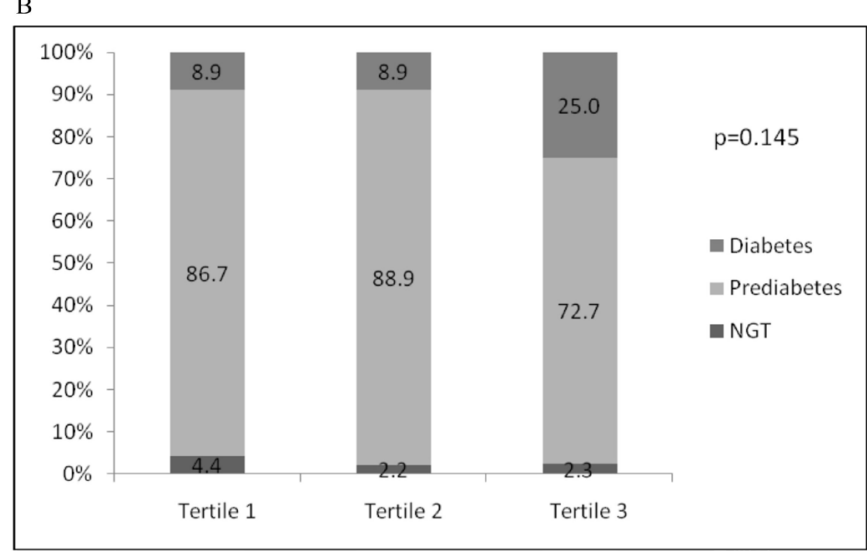

Figure 2 (A) Prevalence rates (\%) of pre-diabetes and diabetes stratified by tertiles of intrahepatic triglyceride (IHTG) content in metabolically healthy abdominal obesity subjects. (B) Prevalence rates (\%) of pre-diabetes and diabetes stratified by tertiles of IHTG content in metabolically unhealthy abdominal obesity subjects.

significantly related to higher prevalence of NAFLD and serum uric acid levels. Furthermore, table 1 showed that, compared with subjects with MHAO, those with MUAO had significantly increased age, IHTG content, prevalence of NAFLD, systolic and diastolic BP, TG, TC, FPG, 2-h PG, HbAlc, serum uric acid and significantly lower level of HDL-C.

\section{Associations of MHAO, NAFLD and IHTG content with pre- diabetes plus diabetes for all subjects}

Table 2 showed that, for all subjects, both MUAO (vs MHAO) and NAFLD (yes vs no) were independently associated with increased risk of pre-diabetes plus diabetes, and the adjusted ORs (95\% CIs) were 10.90 (3.15 to $37.69, \mathrm{p}<0.001$ ) and 3.02 (1.47 to $6.20, \mathrm{p}=0.003$ ), respectively. Higher IHTG content was significantly associated with increased risk of pre-diabetes plus diabetes with the adjusted OR (95\% CI) of per SD increase of IHTG content of 1.62 ( 1.07 to $2.46, \mathrm{p}=0.024)$. With the tertile 1 of IHTG content as the reference, the tertile 3 showed significantly higher risk of pre-diabetes plus diabetes (adjusted OR (95\% CI): 3.13 (1.28 to 7.61$), \mathrm{p}=0.012$ ). And there was a significantly positive trend of increasing categories of IHTG content tertiles with excessive risk of pre-diabetes plus diabetes (trend test: $\mathrm{p}=0.011$ ). There was no significant interaction between MHAO with either NAFLD or tertiles of IHTG content for risk of pre-diabetes plus diabetes (both $p$ values $>0.05$ ).

Stratified analyses on associations of NAFLD and IHTG content with pre-diabetes plus diabetes by MHAO and MUAO

Multivariable logistic regression analyses stratified by MHAO and MUAO separately were conducted (table 2). For MHAO subjects, NAFLD was independently associated with increased risk of pre-diabetes plus diabetes (adjusted OR (95\% CI) : 2.65 (1.25 to 5.60), $\mathrm{p}=0.011$ ). Per SD increase of IHTG content was marginally associated with excessive risk of pre-diabetes plus diabetes with the adjusted OR (95\% CI) of 1.55 (1.00 to 2.40, p=0.051). Compared with the tertile 1 of IHTG content, both the tertile 2 and tertile 3 groups showed significantly increased risks of pre-diabetes plus diabetes with the adjusted ORs (95\% CI) of 2.31 (1.03 to 5.17, $\mathrm{p}=0.042)$ and 2.81 ( 1.14 to $6.90, \mathrm{p}=0.024)$, respectively. And there was also a significantly positive trend between increasing categories of IHTG content tertiles and excessive risk of pre-diabetes plus diabetes (trend test: $\mathrm{p}=0.021$ ). For MUAO subjects, neither NAFLD nor IHTG content was found to be significantly associated with risk of pre-diabetes plus diabetes.

\section{DISCUSSION}

In the present study of 428 subjects with abdominal obesity, $294(68.7 \%)$ and $134(31.3 \%)$ were identified as MHAO and MUAO, respectively. Both MUAO (vs MHAO) and NAFLD (vs non-NAFLD) were independently associated with increased risks of pre-diabetes plus diabetes. Furthermore, higher IHTG content was significantly associated with increased risk of pre-diabetes plus diabetes, and there was a significantly positive trend between increasing categories of IHTG content tertiles and excessive risks of pre-diabetes plus diabetes. Stratified analyses showed similar results for subjects with MHAO but not for those with MUAO.

The concept of MHO has been established for a subgroup of subjects with obesity who do not exhibit metabolic and cardiovascular complications at a given time point, such as diabetes and atherosclerosis, for a few decades. ${ }^{24}{ }^{25}$ Compared with subjects with MUO, those with $\mathrm{MHO}$ are characterised by lower liver and visceral fat, higher subcutaneous leg fat, greater cardiorespiratory fitness, physical activity and insulin sensitivity, lower levels of inflammation, and normal adipose tissue function. ${ }^{26}$ However, it could be debated whether MHO predicts the risk of diabetes compared with metabolically healthy normal weight or MUO. Hinnouho based on the Whitehall II cohort study found a significantly decreased risk of diabetes for MHO compared with metabolically unhealthy obesity (MUO) (HR=1.98 (MUO vs MHO), 95\% CI 1.39 to 2.83$).{ }^{27}$ The present study found similar results that 


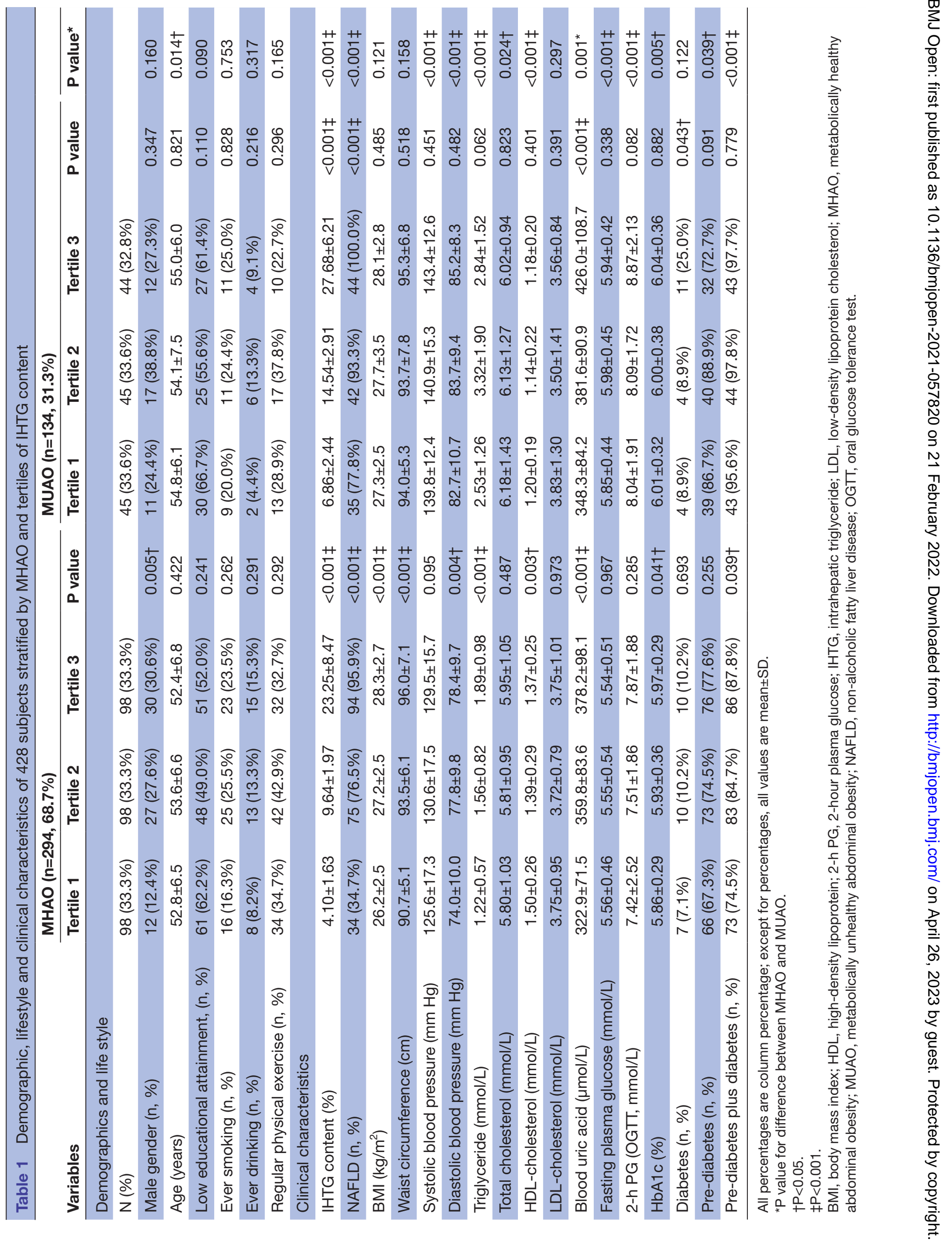


Table 2 Adjusted ORs with associated $95 \% \mathrm{Cl}$ of MUAO, NAFLD and IHTG content for pre-diabetes plus diabetes

\begin{tabular}{|c|c|c|c|}
\hline \multirow[b]{2}{*}{ Variables } & \multicolumn{3}{|c|}{ Pre-diabetes plus diabetes } \\
\hline & OR & $95 \% \mathrm{Cl}$ & $P$ value \\
\hline \multicolumn{4}{|l|}{ All subjects } \\
\hline MUAO versus MHAO & 10.90 & 3.15 to 37.69 & $<0.001^{*}$ \\
\hline NAFLD versus non-NAFLD & 3.02 & 1.47 to 6.20 & $0.003^{*}$ \\
\hline IHTG content $(\%) \dagger$ & 1.62 & 1.07 to 2.46 & $0.024^{*}$ \\
\hline \multicolumn{4}{|l|}{ IHTG content tertiles $\ddagger$} \\
\hline Tertile 1 & 1.00 & & \\
\hline Tertile 2 & 1.81 & 0.86 to 3.81 & 0.117 \\
\hline Tertile 3 & 3.13 & 1.28 to 7.61 & $0.012^{*}$ \\
\hline Trend test & & & $0.011^{*}$ \\
\hline \multicolumn{4}{|l|}{ Interaction test } \\
\hline MUAO*NAFLD & & & 0.956 \\
\hline MUAO^Tertiles of IHTG & & & 0.869 \\
\hline \multicolumn{4}{|l|}{ MHAO subjects } \\
\hline NAFLD versus non-NAFLD & 2.65 & 1.25 to 5.60 & $0.011^{*}$ \\
\hline IHTG content $(\%) \dagger$ & 1.55 & 1.00 to 2.40 & 0.051 \\
\hline \multicolumn{4}{|l|}{ IHTG content tertiles $\ddagger$} \\
\hline Tertile 1 & 1.00 & & \\
\hline Tertile 2 & 2.31 & 1.03 to 5.17 & $0.042^{*}$ \\
\hline Tertile 3 & 2.81 & 1.14 to 6.90 & $0.024^{*}$ \\
\hline Trend test & & & $0.021^{*}$ \\
\hline \multicolumn{4}{|l|}{ MUAO subjects } \\
\hline NAFLD versus Non-NAFLD & 4.77 & 0.07 to 327.48 & 0.469 \\
\hline IHTG content $(\%) \dagger$ & 0.81 & 0.13 to 5.26 & 0.830 \\
\hline \multicolumn{4}{|l|}{ IHTG content tertiles $\ddagger$} \\
\hline Tertile 1 & 1.00 & & \\
\hline Tertile 2 & 3.22 & 0.24 to 43.54 & 0.378 \\
\hline Tertile 3 & 1.90 & 0.15 to 23.69 & 0.620 \\
\hline Trend test & & & 0.558 \\
\hline
\end{tabular}

OR was adjusted for age, sex, educational level, ever smoking, ever drinking, physical activity, BMI, systolic and diastolic BP, triglyceride, total cholesterol, HDL-cholesterol and LDL-cholesterol and serum uric acid.

${ }^{*} \mathrm{P}<0.05$.

TOR and $95 \% \mathrm{Cl}$ were expressed by per SD increase of IHTG content. ‡OR and $95 \% \mathrm{Cl}$ was expressed by the first quartile of IHTG content as the reference.

IHTG, intrahepatic triglyceride; MHAO, metabolically healthy abdominal obesity; MUAO, metabolically unhealthy abdominal obesity; NAFLD, non-alcoholic fatty liver disease.;

MUAO was significantly associated with increased risk of pre-diabetes plus diabetes compared with MHAO but with a much higher adjusted OR $(95 \%$ CI) (10.90 (3.15 to 37.69)). Hinnouho and others further found that MHO showed a significant increased risk of type 2 diabetes mellitus (T2DM) incidence compared with metabolically healthy normal weight. ${ }^{27-29}$ We cannot evaluate the risk of MHAO on diabetes compared with metabolically healthy normal weight since all the subjects in the present study were central obese and none of them could be classified as metabolically healthy or unhealthy normal weight. And because we had a relatively small sample size, we might find the adjusted OR was much higher than those from other. ${ }^{27-29}$

Little evidence is available on differences of prevalence of NAFLD or liver fat content between MHO and MUO. In the present study, we found subjects with MUO, compared with those with MHO, showed significantly higher prevalence of NAFLD $(90.3 \%$ vs $69.1 \%)$ and IHTG content $(16.3 \pm 9.5$ vs $12.3 \% \pm 9.5 \%)$ (both p values $<0.001)$. Our findings indicated the prevalence of NAFLD and IHTG content are still common and high even for those with MHO. Meanwhile, MHO is commonly identified based on the presence of obesity and absence of metabolic syndrome, neither NAFLD nor liver fat content has been considered as an additional criterion when defining and diagnosing MHO. Therefore, our findings implied that screening of NAFLD and intervention to reduce IHTG content for those seemly healthy obese should be strengthened.

Our previous findings showed that NAFLD was significantly associated with increased risk of T2DM prevalence. ${ }^{15}$ The present study expanded the positive association of NAFLD to risk of pre-diabetes plus diabetes for all subjects as well as for those with MHAO, and the adjusted ORs (95\% CI) were 3.02 (1.47 to 6.20) and 2.65 (1.25 to 5.60 ) (both $p$ values $<0.05$ ), respectively. NAFLD has been generally diagnosed by hepatic ultrasonography scanning. In the present study, we conducted IHTG content measurement by using magnetic resonance spectroscopy to quantify the extent of liver fat in these abdominal obese subjects. And we found that IHTG content was significantly associated with increased risk of pre-diabetes plus diabetes with the adjusted OR (95\% CI) of per SD increase of IHTG content of 1.62 (1.07 to 2.46, $\mathrm{p}=0.024$ ). Moreover, we found a significantly positive trend between increasing categories of IHTG content tertiles and excessive risks of pre-diabetes plus diabetes.

Quantitative MRI proton-density fat fraction method has been proved to serve as accurate noninvasive biomarkers for quantifying liver steatosis ${ }^{30}$ and liver fat content was found to be correlated with insulin resistance, ${ }^{31}$ but evidence was scarce on association between the quantity of liver fat and risk of diabetes. Our results on the association between IHTG content and risks of pre-diabetes plus diabetes might account for possibly a novel finding for the present study.

We further conducted stratified analyses on the associations of IHTG content with risk of pre-diabetes plus diabetes for subjects with MHAO and MUAO separately. For those with MHAO, the association of IHTG content with risk of pre-diabetes plus diabetes was marginally significant, and the adjusted OR (95\% CI) of per SD increase of IHTG content was 1.55 (1.00 to $2.40, \mathrm{p}=0.051$ ). With the first tertile of IHTG content as the reference group, the adjusted ORs $(95 \% \mathrm{CI})$ of risks of pre-diabetes plus diabetes for the second and third tertiles were 2.31 (1.03 to 5.17) and 2.81 (1.14 to 6.90) (both $\mathrm{p}$ values $<0.05$ ), 
respectively. The positive trend between increasing categories of IHTG content tertiles and excessive risks of prediabetes plus diabetes was also statistically significant for the subgroup with MHAO (trend test $p$ value $<0.05$ ). Our findings implied that increased IHTG content was associated with excessive risk of pre-diabetes and diabetes even for MHO subjects. To the best of our knowledge, we were probably the first to find the positive associations of IHTG content with risks of diabetes and pre-diabetes for MHAO subjects. The reason for non-significant results for MUAO subgroups may be due to the relatively small sample size of subjects with MUAO ( $\mathrm{n}=134)$.

NAFLD and liver fat quantity has not been currently considered in the definitions and diagnose criteria of $\mathrm{MHO},{ }^{16}{ }^{26}$ although liver is one of the main parts of fat accumulation when obesity occurs. The present study found that around $69 \%$ of subjects with MHAO were diagnosed as NAFLD. Most importantly, even for these apparently MHO individuals, NAFLD and higher IHTG content were both significantly associated with increased risks of pre-diabetes plus diabetes. Therefore, our findings implied that the current criteria of MHO may not be appropriate. NAFLD, quantity of liver fat or abdominal fat content (obtained from ultrasonography or CT-scanning techniques) should be considered as additional criterion when defining and diagnosing MHO if more evidence could be proved in future, especially from the prospective cohort studies with larger sample sizes.

A few limitations of the present study should be recognised when generalising our findings to other populations. First, all subjects were abdominally obese and were not randomly sampled from their living communities; therefore, we could not assess the effect of MHAO as compared with metabolically healthy non-obesity and we might also under-estimate the true associations of MHAO as compared with MUAO on risks of pre-diabetes plus diabetes. Second, the present analyses were based on the baseline information of our ongoing cohort study, therefore, we cannot determine the temporal sequence among MHAO and pre-diabetes plus diabetes. Third, our sample size was small, especially for the MUAO subgroup and we may not have enough power to determine their true associations. On the other hand, we still have some strengths in the present study. For example, we used IHTG content by magnetic resonance spectroscopy, which was relatively measurement of liver fat. And we were probably the first to find the positive associations of IHTG content with risks of diabetes and pre-diabetes, especially for subjects with MHAO.

\section{CONCLUSIONS}

NAFLD were diagnosed in $69 \%$ of MHAO and $90 \%$ of MUAO subjects, and the prevalence rates of pre-diabetes plus diabetes were linearly increased across the tertiles of IHTG content. NAFLD and higher IHTG content were independently associated with increased risks of prediabetes plus diabetes for all subjects as well as for the
MHAO subgroups. Therefore, our findings imply that NAFLD or quantity of liver fat should be considered as additional criterion when defining and diagnosing MHO. Furthermore, screening of NAFLD and intervention to reduce liver fat should be strengthened even for the seemly healthy obese subjects.

\section{Author affiliations}

${ }^{1}$ Lianqian Community Health Service Center, The First Affiliated Hospital of Xiamen University, School of medicine, Xiamen university, Xiamen, China

${ }^{2}$ Department of Radiology, The First Affiliated Hospital of Xiamen University, School of medicine, Xiamen university, Xiamen, China

${ }^{3}$ Department of Endocrinology, Xiamen Branch, Zhongshan Hospital, Fudan University, Xiamen, China

${ }^{4}$ Department of Nursing, the First Affiliated Hospital of Xiamen University, School of medicine, Xiamen university, Xiamen, China

${ }^{5}$ Xiamen Diabetes Institute, The First Affiliated Hospital of Xiamen University, School of medicine, Xiamen university, Xiamen, China

${ }^{6}$ Department of Endocrinology and Diabetes, the First Affiliated Hospital of Xiamen University, School of medicine, Xiamen university, Xiamen, China

${ }^{7}$ Xiamen Institute of Cardiovascular Diseases, the First Affiliated Hospital of Xiamen University, School of medicine, Xiamen university, Xiamen, China

${ }^{8}$ Key laboratory of Neonatal Diseases, Children's Hospital of Fudan University Xiamen Branch \& Xiamen Children's Hospital, Xiamen, China

${ }^{9}$ Fujian Province Key Laboratory of Diabetes Translational Medicine, the First Affiliated Hospital of Xiamen University, School of medicine, Xiamen university, Xiamen, China

${ }^{10}$ Epidemiology Research Unit, Translational Medicine Research Center, the First Affiliated Hospital of Xiamen University, School of medicine, Xiamen university, Xiamen, China

Acknowledgements This study was completed with the assistance of the Lianqian community health service center, Xiamen, China. And we are grateful to all the subjects for their participation.

Contributors QX, JZ, HH, NC and ZL performed the statistical analysis and wrote the manuscript. $\mathrm{HH}, \mathrm{FL}, \mathrm{YL}, \mathrm{ML}$ and $\mathrm{CH}$ participated in the data collection. SW and WZ contributed to discussion. CL, NC, SW and JZ participated in the design of the study and edited the manuscript. CL and ZL are the guarantors of this work and, as such, had full access to all the data in the study and take responsibility for the integrity of the data and the accuracy of the data analysis.

Funding This study was supported by the grants from National Key R\&D Program of China (No. 2017YFC0907100), Natural Science Foundation of China (No. 81870611), Natural Science Foundation of Fujian Province (2019J01569), Open project of State Key Laboratory of Cellular Stress Biology, Xiamen University (№. SKLCSB2019KF004).

\section{Competing interests None declared.}

Patient and public involvement Patients and/or the public were not involved in the design, or conduct, or reporting, or dissemination plans of this research.

\section{Patient consent for publication Not applicable.}

Ethics approval This study was approved by the Human Research Ethics Committee of the First Affiliated Hospital of Xiamen University (Xiamen, China) (number/ID of the obtained ethics approval: 2011YLS-013). Participants gave informed consent to participate in the study before taking part.

Provenance and peer review Not commissioned; externally peer reviewed. Data availability statement Data are available upon reasonable request.

Open access This is an open access article distributed in accordance with the Creative Commons Attribution Non Commercial (CC BY-NC 4.0) license, which permits others to distribute, remix, adapt, build upon this work non-commercially, and license their derivative works on different terms, provided the original work is properly cited, appropriate credit is given, any changes made indicated, and the use is non-commercial. See: http://creativecommons.org/licenses/by-nc/4.0/.

\section{ORCID iDs}

Ning Chen http://orcid.org/0000-0002-8978-7035

Changqin Liu http://orcid.org/0000-0001-8063-7906 


\section{REFERENCES}

1 Saeedi P, Petersohn I, Salpea P, et al. Global and regional diabetes prevalence estimates for 2019 and projections for 2030 and 2045: Results from the International Diabetes Federation Diabetes Atlas, $9^{\text {th }}$ edition. Diabetes Res Clin Pract 2019;157:107843.

2 Zheng Y, Ley SH, Hu FB. Global aetiology and epidemiology of type 2 diabetes mellitus and its complications. Nat Rev Endocrinol 2018;14:88-98.

3 Chatterjee S, Khunti K, Davies MJ. Type 2 diabetes. Lancet 2017;389:2239-51.

4 Legler J, Fletcher T, Govarts E, et al. Obesity, diabetes, and associated costs of exposure to endocrine-disrupting chemicals in the European Union. J Clin Endocrinol Metab 2015;100:1278-88.

5 GBD 2015 Obesity Collaborators, Afshin A, Forouzanfar MH, et al. Health effects of overweight and obesity in 195 countries over 25 years. N Engl J Med 2017;377:13-27.

6 Prospective Studies Collaboration, Whitlock G, Lewington S, et al. Body-mass index and cause-specific mortality in 900000 adults: collaborative analyses of 57 prospective studies. Lancet 2009;373:1083-96.

7 Vague J. The degree of masculine differentiation of obesities: a factor determining predisposition to diabetes, atherosclerosis, gout, and uric calculous disease. Am J Clin Nutr 1956;4:20-34.

8 Sims EA. Are there persons who are obese, but metabolically healthy? Metabolism 2001;50:1499-504.

9 Stefan N, Häring H-U, Hu FB, et al. Metabolically healthy obesity: epidemiology, mechanisms, and clinical implications. Lancet Diabetes Endocrinol 2013;1:152-62.

10 Alberti KGMM, Eckel RH, Grundy SM, et al. Harmonizing the metabolic syndrome: a joint interim statement of the International Diabetes Federation Task Force on Epidemiology and Prevention; National Heart, Lung, and Blood Institute; American Heart Association; World Heart Federation; International Atherosclerosis Society; and International Association for the Study of Obesity. Circulation 2009;120:1640-5.

11 Zhao Y, Qin P, Sun H, et al. Metabolically healthy general and abdominal obesity are associated with increased risk of hypertension. Br J Nutr 2020;123:583-91.

12 Bell JA, Kivimaki M, Hamer M. Metabolically healthy obesity and risk of incident type 2 diabetes: a meta-analysis of prospective cohort studies. Obes Rev 2014;15:504-15.

13 Targher G, Byrne CD. Clinical review: nonalcoholic fatty liver disease: a novel cardiometabolic risk factor for type 2 diabetes and its complications. J Clin Endocrinol Metab 2013;98:483-95.

14 Yan B, Shi X, Zhang $\mathrm{H}$, et al. Association of serum irisin with metabolic syndrome in obese Chinese adults. PLoS One 2014;9:e94235

15 Li Z, Lin M, Liu C, et al. Fetuin-B links nonalcoholic fatty liver disease to type 2 diabetes via inducing insulin resistance: association and path analyses. Cytokine 2018;108:145-50.
16 Zhang J, Xu Q, Lai F, et al. Joint associations of metabolically healthy abdominal obesity and non-alcoholic fatty liver disease with prediabetes and diabetes in Chinese adults. BMJ Open Diabetes Res Care 2021;9:e002362.

17 Jian-gao F, Chinese Liver Disease Association. Guidelines for management of nonalcoholic fatty liver disease: an updated and revised edition. Zhonghua Gan Zang Bing Za Zhi 2010;18:163-6.

18 Wang D, Liu Y, Liu S, et al. Serum fetuin-B is positively associated with intrahepatic triglyceride content and increases the risk of insulin resistance in obese Chinese adults: a cross-sectional study. $J$ Diabetes 2018;10:581-8.

19 Frimel TN, Deivanayagam S, Bashir A, et al. Assessment of intrahepatic triglyceride content using magnetic resonance spectroscopy. J Cardiometab Syndr 2007;2:136-8.

20 Zhou B-F, Cooperative Meta-Analysis Group of the Working Group on Obesity in China. Cooperative Meta-Analysis Group of the Working Group on Obesity in C. Predictive values of body mass index and waist circumference for risk factors of certain related diseases in Chinese adults-study on optimal cut-off points of body mass index and waist circumference in Chinese adults. Biomed Environ Sci 2002;15:83-96.

21 Alberti KGMM, Zimmet P, Shaw J, et al. The metabolic syndrome-a new worldwide definition. Lancet 2005;366:1059-62.

22 Fingeret M, Marques-Vidal P, Vollenweider P. Incidence of type 2 diabetes, hypertension, and dyslipidemia in metabolically healthy obese and non-obese. Nutr Metab Cardiovasc Dis 2018;28:1036-44.

23 American Diabetes Association. Classification and diagnosis of diabetes: standards of medical care in diabetes-2020. Diabetes Care 2020;43:S14-31.

24 Stefan N, Häring $\mathrm{H}-\mathrm{U}$, Schulze MB. Metabolically healthy obesity: the low-hanging fruit in obesity treatment? Lancet Diabetes Endocrinol 2018;6:249-58.

25 Stefan N, Kantartzis K, Machann J, et al. Identification and characterization of metabolically benign obesity in humans. Arch Intern Med 2008;168:1609-16.

26 Blüher M. Metabolically healthy obesity. Endocr Rev 2020;41:405-20.

27 Hinnouho G-M, Czernichow S, Dugravot A, et al. Metabolically healthy obesity and the risk of cardiovascular disease and type 2 diabetes: the Whitehall II cohort study. Eur Heart J 2015;36:551-9.

28 Wei Y, Wang J, Han X, et al. Metabolically healthy obesity increased diabetes incidence in a middle-aged and elderly Chinese population. Diabetes Metab Res Rev 2020;36:e3202.

29 Gaiță D, Moșteoru S. Metabolically healthy versus unhealthy obesity and risk for diabetes mellitus and cardiovascular diseases. Cardiovasc Endocrinol 2017;6:23-6.

30 Kramer H, Pickhardt PJ, Kliewer MA, et al. Accuracy of liver fat quantification with advanced $\mathrm{CT}, \mathrm{MRI}$, and ultrasound techniques: prospective comparison with MR spectroscopy. AJR Am J Roentgenol 2017;208:92-100.

31 Dong Z, Luo Y, Cai $\mathrm{H}$, et al. Noninvasive fat quantification of the liver and pancreas may provide potential biomarkers of impaired glucose tolerance and type 2 diabetes. Medicine 2016;95:e3858. 